\title{
THE PLASTIC RESPONSE TO MONOCULAR DEPRIVATION PERSISTS IN KITTEN VISUAL CORTEX AFTER CHRONIC DEPLETION OF NOREPINEPHRINE ${ }^{1}$
}

\author{
MARK F. BEAR ${ }^{2}$ and J. D. DANIELS \\ Center for Neural Science and Division of Engineering, Brown University, Providence, Rhode Island 02912
}

Reccived July 20, 1982; Revised September 7, 1982; Accepted September 15, 1982

\begin{abstract}
In order to clarify the role of norepinephrine (NE) in visual cortical plasticity, we monocularly deprived kittens that had received systemic injections of the neurotoxin 6-hydroxydopamine (6OHDA) shortly after birth. We found, using high pressure liquid chromatography, that this means of drug treatment produces a permanent and substantial reduction in the level of cortical $\mathrm{NE}$ as compared with littermate controls. Nonetheless, single unit recording in area 17 of these kittens revealed no difference in the cortical response to monocular deprivation: both drug-treated and control kittens displayed large ocular dominance shifts to the open eye.

Because local depletion of NE by intracortical 6-OHDA in kittens can prevent the expected ocular dominance shift after short-term monocular deprivation, we propose that neocortex has the capacity to compensate for chronic depletion of $\mathrm{NE}$ in a way which allows for the possibility of plastic changes.
\end{abstract}

Several features of the noradrenergic innervation of the cerebral neocortex have focused interest on its role in cortical development and plasticity. First, the noradrenergic axons from the locus ceruleus (LC) are likely to be the first extrinsic input to the cortical mantle, arriving well before birth (Levitt and Moore, 1979). Second, the tangential organization of this projection and the widespread collateral distributions of single LC axons allow the simultaneous release of norepinephrine (NE) in vast cortical fields (Morrison et al., 1981). Third, converging anatomical (Descarries et al., 1977) and biochemical (Reader et al., 1976) evidence suggest that NE can evoke a physiological response at sites distant from the point of release. Finally, the physiological action of NE in the neocortex is largely mediated by $\beta$ receptors (Minneman et al., 1979) and ultimately leads to an increase in the

\footnotetext{
1 This research was supported by United States Office of Naval Research Contract N0014-81-K-0136. We gratefully acknowledge Dr. R. L. Patrick for use of the high pressure liquid chromatography and for helpful suggestions regarding the biochemistry; Lederle Labs for supplying Flaxedil; M. Schwartz, M. Evens, S. Nelson, and S. A. Bianco for technical assistance; and T. Kasamatsu, M. Paradiso, M. Katz, D. Berson, and C. Hohmann for helpful criticism of the manuscript. Professors L. N Cooper and F. F. Ebner also aided our progress with helpful discussions. We thank Paula Kanazawich for typing the manuscript.

${ }^{2}$ To whom correspondence should be addressed at Box G, Division of Biology and Medicine, Brown University, Providence, RI 02912
}

level of cyclic adenosine monophosphate (cAMP) in the target neurons. The exact consequences of increasing intracellular levels of this cyclic nucleotide in cortical neurons are not well understood, but cAMP could play a major role in cytodifferentiation, both in developing and mature cells (McMahon, 1974).

Striate cortex, area 17, of the cat has proven to be a valuable system for the study of postnatal cortical development. This system offers several important advantages. First, the normal organization of adult striate cortex is relatively well understood and a strong data base exists on the postnatal development of the physiological properties of visual cortical neurons. Second, highly reproducible physiological and anatomical changes may be induced by simple environmental deprivation paradigms, such as monocular lid closure, during a critical period that extends from approximately 3 weeks to 3 months of age (Hubel and Wiesel, 1970). Finally, the functional connectivity of striate cortex may be conveniently measured with the physiological assay of ocular dominance introduced by Hubel and Wiesel (1962). Using this system, Kasamatsu, Pettigrew, and co-workers have investigated the contribution of the locus ceruleus to visual cortical plasticity. They have employed the pharmacological agent, 6-hydroxydopamine (6-OHDA), a neurotoxin relatively specific for catecholamine-containing nerve terminals (Ungerstedt, 1968; Bloom et al., 1969; Uretsky and Iverson, 1970). These authors report that 
the depletion of cortical NE in kittens by either intraventricular (Kasamatsu and Pettigrew, 1979) or intracortical (Kasamatsu et al., 1979) administration of 6-OHDA prevents the ocular dominance shift that normally results from monocular deprivation during the critical period. Moreover, when NE is replaced by continous microperfusion, the cortex will again respond normally to environmental modification (Kasamatsu et al., 1979). These results led Kasamatsu and Pettigrew to hypothesize that the normal catecholaminergic innervation of cerebral cortex is required for developmental plasticity.

It is now clear that several mechanisms, acting independenlly or in concert, can account for a shifted ocular dominance histogram after monocular deprivation (see Movshon and Van Sluyters, 1981, for review). These include competition for synaptic territory by geniculate fibers (Guillery, 1972; Hubel et al., 1977) and intracortical suppression of the deprived eye responses (Kratz et al., 1976). Which mechanism predominates depends critically on the length of deprivation and the age at which deprivation is initiated (LeVay et al., 1980). An important step in defining the role of the noradrenergic innervation in visual cortical plasticity is to identify which of these mechanisms require NE. Unfortunately, this inquiry has been confounded by limitations in the techniques employed to deplete catecholamines. For example, the intraventricular dose of 6-OHDA required to deplete adequately cortical NE causes severe behavioral side effects such as sham rage, compulsive turning, and even grand mal seizures (Kasamatsu and Pettigrew, 1979). Although these problems are avoided when 6-OHDA is applied intracortically by a minipump-cannula system (Kasamatsu, et al., 1979), the NE depletion produced by this technique is local and not uniform (Kasamatsu et al., 1981a). This restricts neurophysiological analysis to a limited sphere of depleted tissue surrounding the cannula site and also complicates the biochemical confirmation of catecholamine depletion. Furthermore, both techniques limit the onset of monocular deprivation to ages when the surgical implantation of cannulae in the cortex is feasible.

An alternative means to deplete uniformly cortical norepinephrine from birth has been well documented in rodents; namely, the systemic injection of neonates with 6-OHDA (Clark et al., 1972; Sachs and Jonsson, 1975). During the 1st postnatal week, the drug has access to the entire nervous system since the blood-brain barrier is incompletely formed at this age (Sachs, 1973). This treatment in rats leads to a pronounced and irreversible depletion of cortical NE. In contrast, the noradrenergic innervation of subcortical structures rapidly regenerates (Sachs and Jonsson, 1975). We elected to try this paradigm for catecholamine depletion in kittens in an attempt to test further the idea that $\mathrm{NE}$ is required for visual cortical plasticity.

In this paper we provide biochemical evidence that the cerebral cortex of kittens can be drastically depleted of norepinephrine with neonatal injections of 6-OHDA. In addition, we present physiological results indicating that visual cortex depleted of $\mathrm{NE}$ by this technique will nonetheless respond normally to monocular deprivation during the critical period. These results, considered with those of Kasamatsu and Pettigrew, suggest that compensation for the lost NE may occur in kittens chronically depleted from birth.

\section{Materials and Methods}

Drug treatment and lid closure. A total of six litters of kittens (21 animals) from our quarantined colony of wildtype queens was used in this study (Table I). Approxi-

TABLE I

Animal history

\begin{tabular}{|c|c|c|c|c|c|}
\hline Litter No. & Animal & Drug Treatment & Visual Deprivation & Age of Recording & Comment \\
\hline & & $\begin{array}{l}m g \text { 6-OHDA/ } \\
\text { hg body weight }\end{array}$ & & days & \\
\hline \multirow[t]{2}{*}{1} & K 113 & $1 \times 100$ & M.D. ${ }^{a} 7$ days & 39 & pilot \\
\hline & $\mathrm{K} 114$ & $-b$ & M.D. 7 days & 41 & pilot \\
\hline \multirow[t]{4}{*}{2} & K 115 & $2 \times 100$ & M.D. 10 days & 42 & - \\
\hline & K 116 & - & M.D. 10 days & 44 & - \\
\hline & K 117 & $2 \times 150$ & & 一 & died, $\mathrm{PND}^{\circ} 2$ \\
\hline & K 118 & $2 \times 300$ & & - & died, PND 2 \\
\hline \multirow[t]{5}{*}{3} & K 119 & $2 \times 200$ & M.D. 10 days & 36 & - \\
\hline & $\mathrm{K} 120$ & - & M.D. 10 days & 39 & - \\
\hline & K 121 & $2 \times 100$ & & - & died, PND 29 \\
\hline & K 122 & - & M.D. 10 days & 45 & - \\
\hline & K 123 & $2 \times 300$ & M.D. 10 days & 47 & - \\
\hline \multirow[t]{3}{*}{4} & K 124 & $2 \times 200$ & M.D. from birth & - & died, PND 17 \\
\hline & K 125 & $2 \times 200$ & M.D. from birth & - & died, PND 95 \\
\hline & K 126 & - & & - & - \\
\hline \multirow[t]{4}{*}{5} & K 127 & $2 \times 200$ & M.D. from birth & 54 & - \\
\hline & K 128 & $2 \times 200$ & M.D. 10 days & 46 & - \\
\hline & K 129 & - & M.D. from birth & 57 & - \\
\hline & K 130 & - & M.D. 10 days & 49 & - \\
\hline \multirow[t]{3}{*}{6} & K 131 & $2 \times 200$ & M.D. 10 days & 36 & - \\
\hline & K 132 & - & M.D. 10 days & 38 & - \\
\hline & K 133 & $2 \times 200$ & M.D. 10 days & 40 & - \\
\hline
\end{tabular}

"M.D., monocular deprivation.

"- none.

${ }^{c}$ PND, postnatal day. 
mately half of the animals in each litter received intraperitoneal injections of 6-OHDA/HCl (Sigma Chemical Co.) dissolved in saline with $0.1 \%$ ascorbate. The remaining animals received vehicle injections and served as controls. All injections were performed blind, such that at the time of physiological recording, the investigators had no knowledge of the animal's drug history. It was found after several pilot animals that the most effective dose was $200 \mathrm{mg}$ of $6-\mathrm{OHDA} / \mathrm{kg}$ of body weight, injected twice with a 24-hr interval on postnatal days 1 and 2 (Table I). After each injection, the kittens were returned to their mothers for normal rearing.

All kittens but four received monocular lid sutures under anesthesia (ketamine/acepromazine) 10 days before the neurophysiological recording session. Ages at the time of lid closure ranged from 26 to 39 days (Table I). The remaining four animals were monocularly deprived from shortly after birth. Sutured lids were inspected daily and any windows were promptly repaired.

Physiological preparation. Animals were prepared for extracellular recording by standard techniques (Daniels et al., 1978). Ketamine/acepromazine (25 and $3 \mathrm{mg} / \mathrm{kg}$, respectively)-anesthesized kittens received tracheal intubation, venous cannulation, scalp incision, and craniotomy. The animals were secured in a modified stereotaxic frame, $114 \mathrm{~cm}$ from a tangent screen. Paralysis was maintained by a continuous infusion of $12 \mathrm{mg} / \mathrm{kg} / \mathrm{hr}$ of gallamine triethiodide (Flaxedil) and artificial respiration of $70 \%$ nitrous oxide, $28 \%$ oxygen, and $2 \% \mathrm{CO}_{2}$ maintained anesthesia throughout the experiment. EEG, body temperature, heart rate, and expired $\mathrm{CO}_{2}$ levels were monitored continuously.

Pupils were dilated with atropine and contact lenses were fitted to provide a slight positive correction and to prevent corneal drying. Optic discs were viewed with an ophthalmoscope and two observers marked the approximate projection of these landmarks onto the tangent screen. For the age of the kittens we worked with, a $20^{\circ}$ nasal and $10^{\circ}$ inferior standard was employed to mark the respective area centralae (Olson and Freeman, 1980).

Single unit recording. Tungsten-in-glass microelectrodes were fitted into our dual microdrive advance which allows simultaneous recording from both hemispheres. Each electrode was positioned over the area centralis representation in area 17 and was passed anteriorly and ventrally down the crown and medial wall of the postlateral gyrus. Extracellular action currents were monitored through a variable filter system producing optimal signal-to-noise ratios for audio detection of cell discharges. Single unit data were collected from each hemisphere approximately every $100 \mu \mathrm{m}$.

Slit and spot visual stimuli, projected by independent systems onto the tangent screen, were manipulated by two joystick controllers. The length, width, and orientation of the slit stimulus and the diameter of the spot were variable. A foot switch controlled a shutter in each projector. With these stimuli, receptive fields of visually responsive cells within $5^{\circ}$ of the horizontal meridian and within $20^{\circ}$ of the vertical meridian were plotted and ocular dominance discriminations were made according to the criteria introduced by Hubel and Wiesel (1962). In addition, the disparity of binocular cells, on-off properties, and speed preferences were noted. Neuronal selec- tivity for the direction of an oriented bar moved across the receptive field was classified as either aspecific, immature, or specific according to criteria modified from Fregnac and Imbert (1978).

Following the 36 -hr recording session, the animals were sacrificed by a lethal intravenous dose of potassium tartrate followed by perfusion through the ascending aorta with saline. The brains were rapidly dissected and samples taken for biochemical analysis were frozen on powdered dry ice. Tissue samples always included the cerebellum and the visual cortex from one hemisphere. The cortical sample included areas 17, 18, and 19, and had a mean wet weight of $1.46 \mathrm{gm}( \pm 0.05 \mathrm{gm}$, standard error). These tissues were stored overnight in a freezer at $-30^{\circ} \mathrm{C}$ until the catecholamine purification procedure could be performed. The remaining hemisphere was preserved in $10 \%$ formaldehyde containing $30 \%$ sucrose for histological analysis.

Biochemistry and histology. Tissue catecholamines were measured by high pressure liquid chromatography (HPLC) with electrochemical detection. Samples were weighed, homogenized in $0.1 \mathrm{M}$ perchloric acid containing $0.1 \mathrm{~mm}$ EDTA, and centrifuged to remove denatured protein. Dihydroxybenzylamine, a catecholamine with a different retention time in the HPLC system than any of the naturally occurring catechols, was added to the perchloric acid supernatant as a standard to monitor recovery. Catechol compounds in the supernatant were then purified by a batch aluminum oxide chromatography technique, based on a column procedure described by Anton and Sayre (1962). An aliquot of the aluminapurified sample was applied to a high pressure liquid chromatography column (Biophase ODS, $5 \mu \mathrm{m}$; Bioanalytical Systems, Inc.), which separates, in order of increasing retention time, norepinephrine, dihydroxybenzylamine, and dopamine from each other. The catechols were eluted off the column with a $0.1 \mathrm{M}$ monobasic sodium phosphate buffer containing $0.13 \mathrm{~mm}$ sodium octyl sulfate, $0.1 \mathrm{~mm}$ EDTA, and 4\% methanol. Buffer $\mathrm{pH}$ was adjusted to 2.6 with phosphoric acid. The electrochemical detector oxidized the catechol compounds and the subsequent currents were measured (Keller et al., 1976). Tissue concentrations of norepinephrine and dopamine were calculated and expressed as nanograms of compound per gram of tissue, wet weight. For each HPLC run, a "least detectable" NE amount was computed from the recovery values.

Formalin-fixed hemispheres were cut on a freezing microtome in the coronal plane at $50 \mu \mathrm{m}$. Sections were mounted on glass slides, hydrated, stained with cresyl violet acetate, dehydrated, cleared in xylene, and coverslipped. Electrode tracks and marker lesions were reconstructed. Any units obtained from adjacent area 18 were discarded from ocular dominance histograms.

\section{Results}

\section{Biochemistry}

The biochemical results shown in Figure 1 clearly confirm that neonatal administration of 6-OHDA is effective in depleting the cortical catecholamines in kittens. On the left side of Figure 1, the tissue concentrations of catecholamines, expressed in nanograms per gram of 


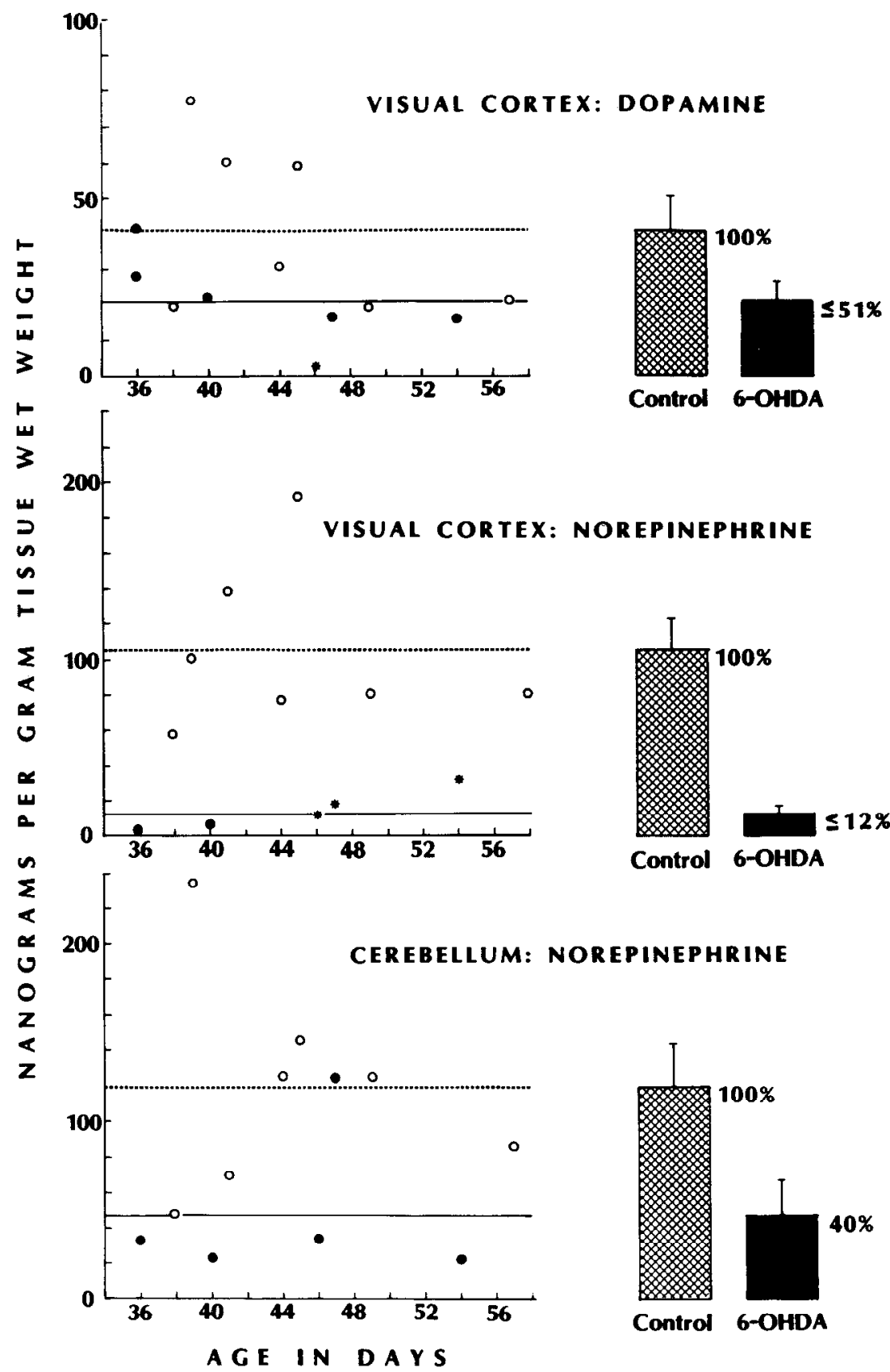

Figure 1. Catecholamine levels in cortex and cerebellum after neonatal 6-OHDA. On the left side, the tissue content of catecholamines is plotted against the age of each kitten at the time of sacrifice. Open circles represent control kittens and closed circles represent 6-OHDA-treated kittens. Catecholamines were not detected in some tissues of drugged kittens and in these cases asterisks indicate the least detectable amount. The dashed lines show the control means; the solid lines illustrate the drug means. These data are recast in the histograms on the right side. Particularly, note the degree to which visual cortical norepinephrine is depleted after neonatal 6-OHDA.

tissue wet weight, are plotted against the age of the animal at the time of sacrifice for both treated and control animals. No clear age-dependent changes in cortical dopamine (DA), cortical norepinephrine, or cerebellar norepinephrine are seen in either group of kittens in the age window we have examined. These data are recast in the histograms on the right side of Figure 1 and also appear in Table II.

Visual cortex: norepinephrine (Fig. 1, middle panel). Drug-treated animals with detectable levels of NE had less than $5 \%$ of control cortical norepinephrine levels. In several kittens, NE was not detected in the cortex at all, but least detectable amounts were relatively high (Fig. 1, asterisks). These points raise the calculated mean level of cortical $\mathrm{NE}$ in drug-treated animals to less than or equal to $12 \%$ of control. The control mean of cortical NE $(105 \pm 17 \mathrm{ng} / \mathrm{gm})$ agrees well with that reported in the literature (Kasamatsu et al., 1981a) for kitten visual cortex.

Visual cortex: dopamine (Fig. 1, upper panel). While the cortical NE was at least $88 \%$ depleted in 6-OHDAtreated kittens, cortical DA levels were as high as $51 \%$ of 
control. This is biochemical support for the existence of a separate dopaminergic projection onto feline visual cortex (Tork and Turner, 1981). As reported previously in the cat (Kasamatsu, et al., 1981a), dopaminergic terminals are notably less susceptible to 6-OHDA than are noradrenergic terminals. In fact, two 36-day-old, drugtreated kittens (K 119 and K 131) had cortical DA in the normal range despite the over $95 \%$ depletion of NE.

Cerebellum: norepinephrine (Fig. 1, lower panel). NE levels in the cerebellum were measured as an index of the extracortical effects of the 6-OHDA treatment. In rats, Kostrzewa and Garey (1976) found that after an

TABLE II

Biochemistry summary

\begin{tabular}{lccc}
\hline Animal & Cortical NE & Cortical DA & Cerebellar NE \\
\hline Control & \multicolumn{3}{c}{$n g / g m$} \\
K 114 & 138 & 60 & 70 \\
K 116 & 77 & 30 & 125 \\
K 120 & 111 & 78 & 235 \\
K 122 & 192 & 59 & 146 \\
K 129 & 81 & 21 & 86 \\
K 130 & 80 & 19 & 125 \\
K 132 & 58 & 19 & 50 \\
X \pm SEM & $105 \pm 17$ & $41 \pm 9$ & $120 \pm 23$ \\
6 -OHDA & & & \\
K 119 & $\leq 1$ & 28 & NA \\
K 123 & $\leq 17^{b}$ & 17 & 124 \\
K 127 & $\leq 32^{b}$ & 16 & 23 \\
K 128 & $\leq 11^{b}$ & $\leq 2^{b}$ & 34 \\
K 131 & 4 & 41 & 33 \\
K 133 & 7 & 22 & 24 \\
X \pm SEM & $\leq 12 \pm 5$ & $<21 \pm 5$ & $48 \pm 19$ \\
\hline
\end{tabular}

"NA, value not available.

${ }^{b}$ Least detectable amount. initial depletion of less than $50 \%$ at 2 weeks postnatal, the cerebellar NE content in 6-hydroxydopa-treated animals increased to over $150 \%$ of control levels by 8 weeks of age. There is no evidence for such a recovery in the cerebellum of the drug-treated kittens. In the age window we have studied, from 5 to 8 weeks of age, the cerebellar NE in 6-OHDA-treated kittens is only $40 \%$ of control.

\section{Behavioral observations}

Kittens treated with 6-OHDA by intraventricular injections suffer from severe behavioral side effects such as sham rage, seizures, and incontinence as well as aphagia and loss of body weight (Kasamatsu and Pettigrew, 1979). Although many of these side effects are eliminated when 6-OHDA is delivered intracortically by a minipump-cannula system, drug-treated animals are nevertheless noticeably listless and exhibit substantial weight loss (personal observation). In Figure 2, body weight is plotted against age for kittens recorded in the present study. It appears that the rate of weight gain in kittens treated with 6-OHDA at birth falls within the normal range. Moreover, simple tests for visuomotor reflexes and casual behavioral observations revealed no difference between experimental and control kittens.

Despite the lack of behavioral manifestations of neonatal 6-OHDA treatment, drugged animals did show an unusually high mortality rate (over $35 \%$ ). Although two animals died within 2 days of the injection, one died unexpectedly after 1 month and another was over 3 months of age at the time of death. Postmortem examination of these animals uncovered no obvious pathology.

Single unit recording after monocular deprivation Monocular deprivation from the time of eye opening. Figure 3 illustrates ocular dominance data from a pair of

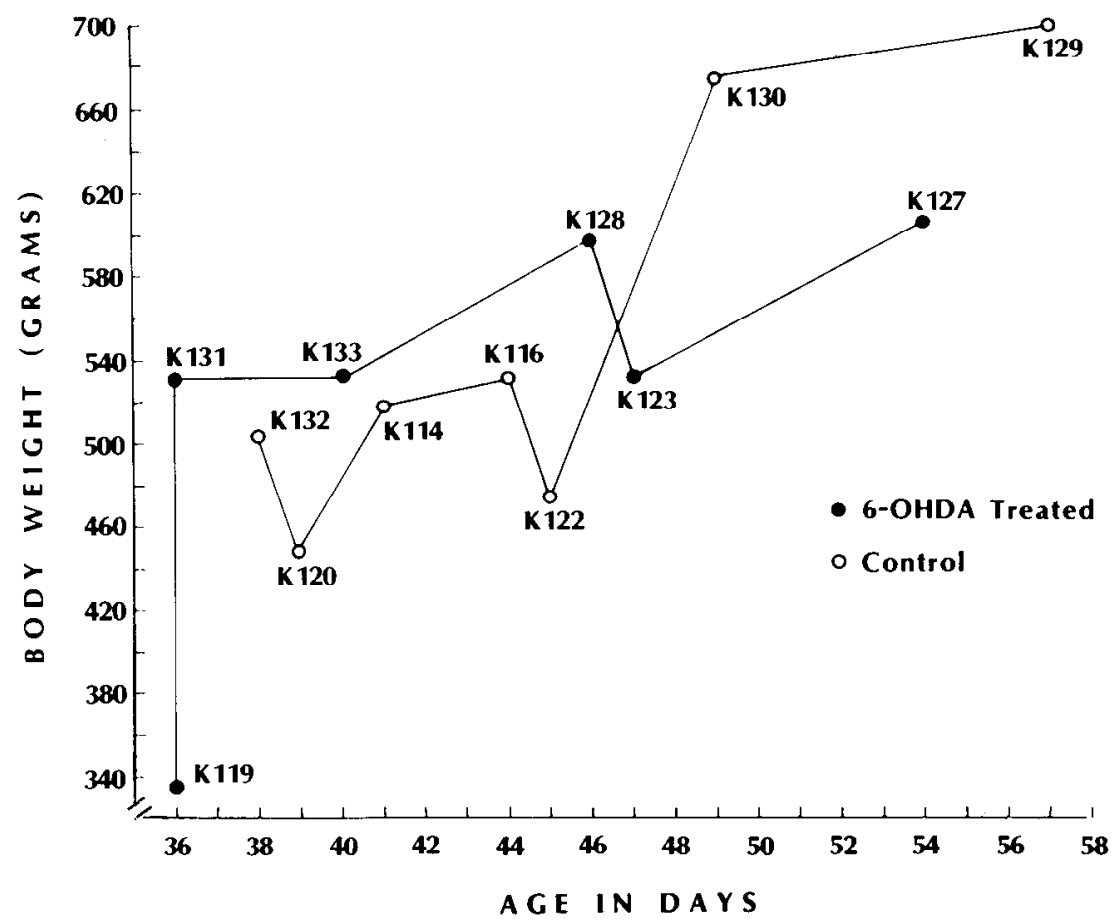

Figure 2. A plot of body weight against age for both groups of kittens. Drug-treated animals (O) show a normal rate of weight gain as do control kittens $(O)$. This observation is consistent with the virtual absence of behavioral side effects in the drug-treated animals. 


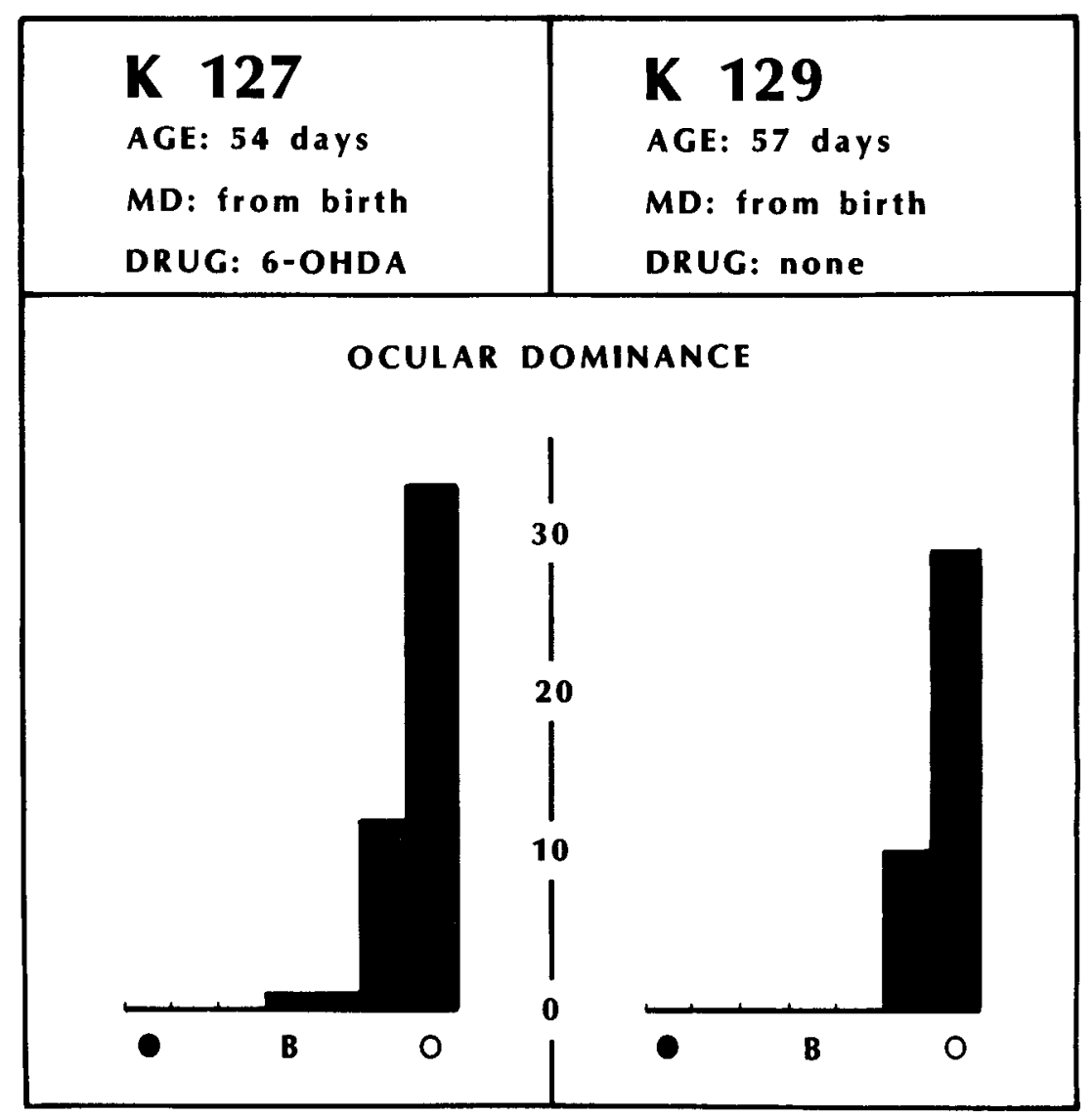

Figure 3. Ocular dominance histograms from a pair of littermates monocularly deprived from just after birth. K 127 received neonatal injections of 6-OHDA; K 129 was injected with vehicle. The number of cells in each of the seven ocular dominance groups are illustrated. The open circle is under the open eye group, the solid circle is under the closed eye group, and the $B$ labels the strictly binocular group (group 4). A strong shift toward the open eye is observed in both kittens.

littermates, monocularly deprived from just after birth. Each histogram is a composite of data recorded from both hemispheres. $\mathrm{K} 127$ received intraperitoneal injections of 6 -OHDA $(200 \mathrm{mg} / \mathrm{kg})$ on postnatal days 1 and 2 . $\mathrm{K} 129$ served as the vehicle-injected control. We recorded from these kittens within 3 days of each other, on postnatal days 54 and 57 , respectively. Both histograms display a large ocular dominance shift to the open eye and no significant difference with regard to binocularity is observed between the two. Other neuronal response properties such as direction selectivity and receptive field size were also similar in these two animals. Biochemical analysis of K 127 confirmed the depletion of cortical NE by the 6-OHDA treatment ('Table II).

Monocular deprivation for 10 days prior to recording. Results from a representative pair of littermates (K 131 and $K 132$ ) with 10 days of monocular deprivation appear in Figure 4. Again, both animals had a shifted ocular dominance profile. These histograms fall within the range of normal variability found after a 10-day monocular deprivation during the critical period (Olson and Freeman, 1975). HPLC analysis confirmed the effectiveness of the drug treatment (Table II).

Another particularly striking example of ocular dominance plasticity without cortical NE is K 119. Ten days of monocular deprivation caused a complete shift in ocular dominance to the open eye in this kitten (Table III). The subsequent biochemical measurement of cate- chols in the visual cortex indicated that this animal had less than $1 \mathrm{ng}$ of $\mathrm{NE} / \mathrm{gm}$ of tissue (wet weight). This represents a cortical NE depletion of over $99 \%$ of control (Table II).

Neurophysiology summary. The results from all successfully recorded kittens were consistent with these representative animals (Table III). The ocular dominance data from all of the animals in this study are compiled and normalized in Figure 5. Both 6-OHDA and control groups display ocular dominance histograms strongly shifted in favor of the open eye. In addition, we observed no significant differences in other cellular response properties such as direction selectivity or receptive field size. Thus, NE-depleted kittens were indistinguishable from controls on the basis of behavior, physiological plasticity, and neuronal response characteristics.

\section{Discussion}

Neonatal 6-OHDA treatment in kittens. It is well documented that intraperitoneal injections of 6-OHDA $(100 \mathrm{mg} / \mathrm{kg})$ in newborn rats will result in a permanent $90 \%$ loss of NE in the cerebral cortex (cf. Sachs and Jonsson, 1975). This procedure usually spares the subcortical projections of the locus ceruleus, and in the cerebellum, NE levels may actually be elevated. These regional differences probably result from the selective destruction of the cells that project to the cortex (Clark et al., 1979). In this study, we provide biochemical evi- 


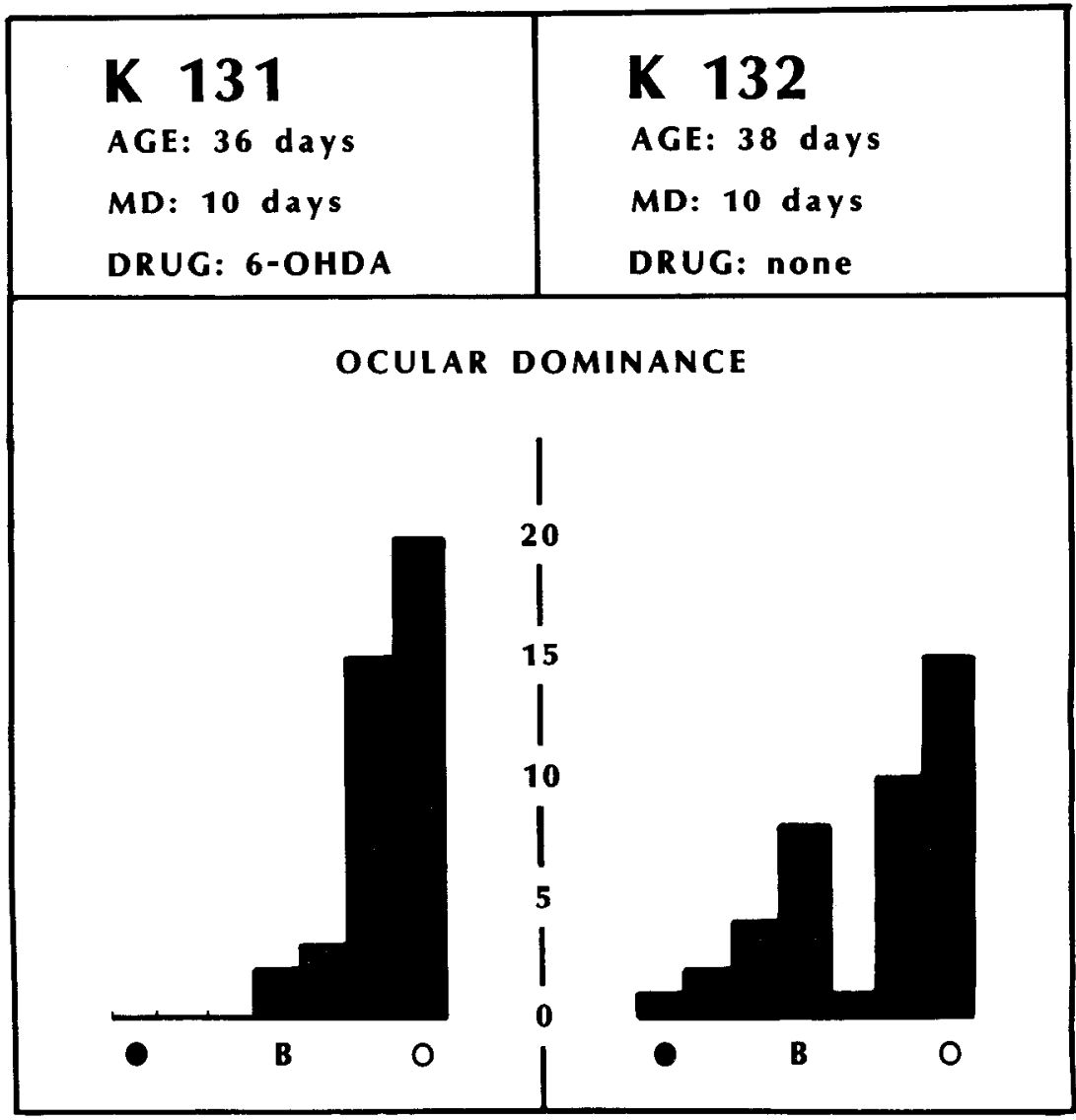

Figure 4. Ocular dominance histograms from a pair of littermates monocularly deprived for the 10 days prior to recording. K 131 received neonatal injections of 6-OHDA; K 132 was injected with vehicle. All conventions are as in Figure 3 . A substantial ocular dominance shift is evident in both animals.

TABLE III

Neurophysiology summary

Induction is an index of the degree to which an ocular dominance histogram shifts after monocular deprivation and is defined as the number of cells dominated by the open eye divided by the cotal number of neurons recorded (after Blakemore, 1978). Binocularity is defined as the number of cells in ocular dominance groups 2 to 6 over the total (after Kasamatsu and Pettigrew, 1979).

\begin{tabular}{lclc}
\hline \multicolumn{1}{c}{ Animal } & No. of Cells & Induction & Binocularity \\
\hline 6 -OHDA & & & \\
K 115 & 37 & 0.81 & 0.46 \\
K 119 & 22 & 1.0 & 0.27 \\
K 127 & 47 & 0.98 & 0.20 \\
K 131 & 40 & 0.95 & 0.50 \\
X & 32 & 0.95 & 0.30 \\
Control & & & \\
K 116 & 40 & 1.0 & 0.27 \\
K 122 & 45 & 0.87 & 0.51 \\
K 129 & 39 & 1.0 & 0.26 \\
K 130 & 38 & 0.66 & 0.39 \\
K 132 & 41 & 0.63 & 0.63 \\
X & 41 & 0.83 & 0.41 \\
\hline
\end{tabular}

dence that neonatal 6-OHDA treatment is also effective in depleting kittens of cortical NE. We note, however, two differences in the way these species respond to drug treatment. First, kittens require a larger dose $(2 \times 200$ $\mathrm{mg}$ of $6-\mathrm{OHDA} / \mathrm{kg}$ of body weight) to achieve the same degree of catecholamine depletion obtained with $100 \mathrm{mg} /$ $\mathrm{kg}$ in rats. Second, the cerebellum of kittens is also

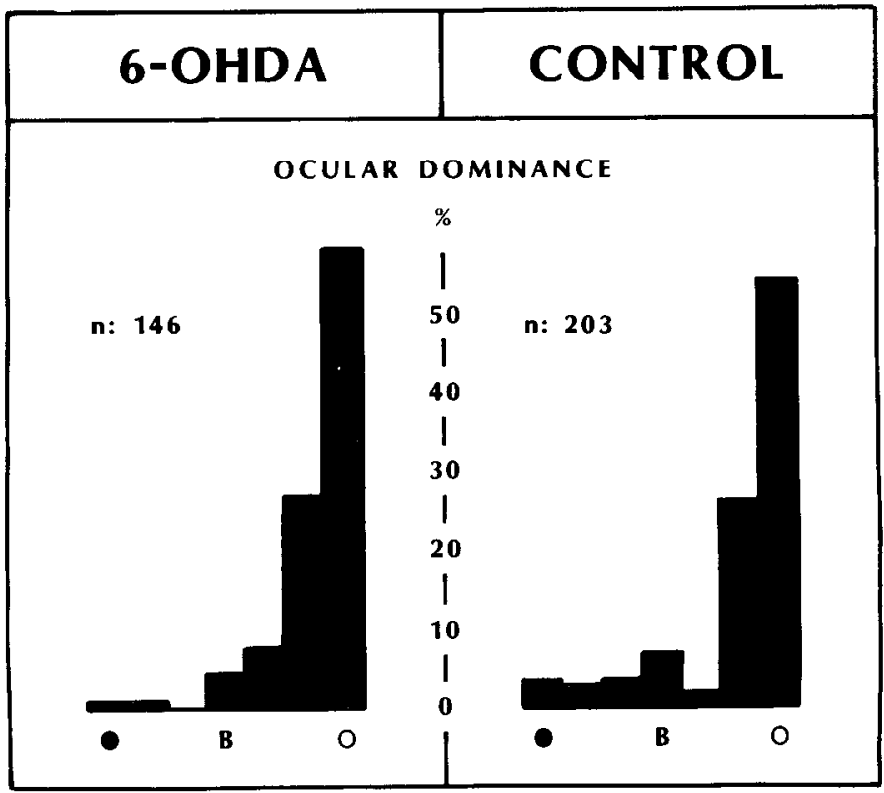

Figure 5. Summary of ocular dominance data obtained from the kittens in this study. Of 146 cells recorded in 6-OHDAtreated kittens, almost $60 \%$ were driven exclusively by the open eye. Conventions are as in Figure 3.

depleted of NE by this procedure. These differences may relate to the anatomical organization of the feline locus ceruleus (Chu and Bloom, 1974). The monoaminergic neurons in the cat brainstem are widely distributed, while 
those in the rat are clustered in discrete nuclei. Thus it is possible that blood-borne 6-OHDA penetrates to different populations of catecholamine-containing neurons in these two species.

While neonatal 6-OHDA treatment results in a virlually complete destruction of the noradrenergic innervation of kitten cerebral cortex, cortical dopamine levels remain remarkably high. This suggests that dopamine plays a role in visual cortex other than simply as a percursor to norepinephrine. Reader et al. (1979) have argued for the existence of a separate dopaminergic projection onto visual cortex and, recently, Tork and Turner (1981) have demonstrated a projection to visual cortex in the cat from dopaminergic cells in the ventromedial tegmentum. Our results indicate that this system is less sensitive to neonatally administered 6-OHDA than is the noradrenergic projection.

There were no obvious differences in the behavior of 6-OHDA-treated and control kittens. We were, therefore, surprised by the high mortality of NE-depleted animals. Since both peripheral and central adrenergic mechanisms play a role in cardiovascular regulation (Doba and Reis, 1974), it is possible that the neonatal 6-OHDA treatment interferes with this vital function. We can only speculate at this point because biochemical measurements of peripheral and brainstem tissues were not made in these kittens.

Effects of monocular deprivation on kitten visual cortex depleted of NE from birth. In our paradigm, we found no evidence that NE depletion alters the cortical response to monocular deprivation. The 6-OHDA-treated kitten monocularly deprived from shortly after birth (K 127) displayed a virtually complete ocular dominance shift toward the open eye, as did the littermate control. To investigate shorter periods of deprivation, we monocularly deprived kittens for 10 days during the critical period. We were guided by Olson and Freeman's (1975) report that this relatively brief period of deprivation will produce a reliable and significant ocular dominance shift in kittens at the height of the critical period. Again, we found that 6-OHDA-treated kittens responded to monocular deprivation as vigorously as did controls: both groups displayed ocular dominance histograms strongly shifted in favor of the open eye. In fact, one 6-OHDAtreated kitten with 10 days of monocular deprivation ( $\mathrm{K}$ 119) had a fully shifted ocular dominance distribution with less than $1 \%$ of control cortical NE. We conclude that physiological plasticity is possible without cortical norepinephrine.

Relation to other studies of cortical development and plasticity after NE removal. Several aspects of cortical development have been studied after neonatal or prenatal destruction of the ascending noradrenergic afferents. In most cases the anatomical changes in cortex have ranged from subtle to nonexistent. For example, Wendlandt et al, (1977) found that neonatal lesions of the locus ceruleus had no effect on the dendritic morphology of cortical neurons. Parnavelas and Blue (1982) report a small difference in the rate of synapse formation (Gray's type I) in rat visual cortex after neonatal 6-OHDA, but only during the 1st postnatal week. It could be argued that because the NE fibers invade the cortical mantle as early as the 16th embryonic day in rats, NE may only be required for aspects of the prenatal differentiation of cortex (Schlumpf et al., 1980). However, the careful study by Lidov and Molliver (1982), in which rat fetuses were systemically administered 6-OHDA, demonstrated no changes in the development of cortical cytoarchitecture, dendritic morphology, or afferent innervation.

Is there a special role for NE that cannot be clearly discerned in such anatomical studies? The provocative results from Kasamatsu's laboratory would suggest that this may be the case. Kasamatsu and colleagues found that while acute 6-OHDA treatment leaves the receptive field properties of visual cortical neurons largely unaffected, the response of kitten visual cortex to monocular deprivation is altered drastically (Kasamatsu and Pettigrew, 1979; Kasamatsu et al., 1979). These authors conclude that NE acts "not by altering patterns of connections directly, but rather by altering the ease with which changes in patterns of connections can be brought about" (Kasamatsu et al., 1981b, p. 265).

The results from the present study appear to contradict Kasamatsu's catecholamine hypothesis of visual cortical plasticity and instead, generally to support the conclusion that NE is not required for normal cortical developmental plasticity. Nevertheless, the phenomenon of decreased sensitivity of visual cortex to sensory deprivation after intracerebral delivery of 6-OHDA has been reproduced by Daw and Rader (1981). Moreover, preliminary results in our own laboratory further confirm the observation that minipump delivery of 6-OHDA to area 17 concurrent with 7 days of monocular deprivation will substantially retard ocular dominance plasticity (Bear et al., 1982). This apparent discrepancy probably stems from the different methods employed to deplete cortical norepinephrine. ${ }^{3}$ In Kasamatsu's paradigm, the drug is administered intracortically, at least 1 month postnatally, and the cortex is depleted acutely (10 days or less before recording). On the other hand, in this study we have administered the 6-OHDA systemically and neonatally and have chronically depleted the visual cortex of $\mathrm{NE}$ (over 1 month before recording). At the moment, more experiments are still required to pinpoint the critical difference between these two paradigms. However, if a critical level of NE is normally required for the appropriate cortical response to deprivation, as Kasamatsu had proposed, then some mechanism must exist to compensate for the chronic loss of cortical NE in kittens treated with 6-OHDA at birth.

Compensation for chronic NE depletion. One compensatory mechanism for chronic denervation is receptor supersensitivity (Cannon and Rosenblueth, 1949). It is well documented in rodents that destruction of the cortical NE fibers will result in an increase in $\beta$-adrenergic receptors postsynaptically (Sporn et al., 1977). Will this

\footnotetext{
${ }^{3}$ Because 6-OHUA depletes cortical DA as well as NE, it could be argued that dopamine, not NE, is the critical factor for plasticity and that it responds differently in the two depletion paradigms. However, the minipump delivery of 6-OHDA and the neonatal 6-OHDA injection are both relatively less effective for destroying DA terminals than NE terminals. Kasamatsu et al. (1981a) report a loss of plasticity with dopamine levels as high as $75 \%$ of control. In our animals, the dopamine level was only $40 \%$ of control. Furthermore, replacement experiments (Kasamatsu et al., 1979) suggest that norepinephrine is the catecholamine required for plasticity.
} 
increase in $\beta$ receptor number compensate for the almost $90 \%$ loss of NE after neonatal 6-OHDA treatment? One in situ index of NE action on $\beta$ receptors in cortex is the measurable blood volume shifts that are provoked by direct cortical stimulation (LaManna et al., 1981). Acute locus ceruleus lesions in rats partially abolish this response. Even when $\beta$ receptor birding is maximal, 2 weeks after unilateral destruction of the locus ceruleus, severe abnormalities in this functional index persist (Harik et al., 1981). These data suggest that receptor proliferation and supersensitivity alone may not be capable of compensating for the loss of cortical NE.

However, Harik et al. (1981) did document a compensatory mechanism independent of receptor supersensitivity. They observed that a normal response of blood volume to cortical stimulation returned 4 weeks after the locus ceruleus lesion. At 4 weeks, NE levels were still only $10 \%$ of control and the specific $\beta$ receptor binding had returned to normal levels. The mechanism for this recovery of function is unknown. These authors speculate that other catecholaminergic systems could sprout to occupy territory vacated by locus ceruleus terminals.

Another possible compensatory mechanism involves interactions with other known transmitter systems in cortex. For example, one input to all of the cerebral cortex releases acetylcholine (Johnston et al., 1981), which at many sites acts in physiological opposition to NE (Krnjevic and Phillis, 1963). Thus, it is conceivable that the functional compensation for the chronic loss of cortical NE could involve a down-regulation of the cortical cholinergic system.

Regardless of the mechanism, the idea of compensation for chronic NE depletion of cortex offers an explanation that is consistent with all of the available data. We propose that a critical amount of norepinephrine is necessary for the normal developmental plasticity of cerebral cortex. At the same time, we speculate that early in development, remarkably adaptive mechanisms exist which can compensate rapidly for the depletion of NE. This idea predicts that many deficits stemming from early NE depletion would only be transient. Indeed, Parnavelas and Blue (1982) have found that the difference in synapse formation in 6 -OHD $\Lambda$-treated neonates lasts only during the 1st postnatal week.

\section{References}

Anton, A. H. and D. F. Sayre (1962) A study of the factors affecting the aluminum oxide-trihydroxy-indole procedure for the analysis of catecholamines. J. Pharmacol. Exp. Ther. 138: 360-375.

Bear, M. F., M. A. Paradiso and J. D. Daniels (1982) Visual cortical plasticity: Deficit after acute, but not chronic, noradrenergic denervation with 6 -hydroxydopamine. Soc. Neurosci. Abstr. 8: 5.11

Blakemore, C. (1978) Maturation and modification in the developing visual system. In Handbook of Sensory Physiology, R. Heid, H. W. Leibowitz, and H. L. Teuver, eds., Vol. 7, pp. 377-426, Springer-Verlag, New York.

Bloom, F. E., S. Algeri, A. Groppetti, A. Revuelta, and E. Costa (1969) Lesions of central norepinephrine terminals with 6$\mathrm{OH}$-dopamine: Biochemistry and fine structure. Science 166 : 1284-1286.

Cannon, W. B., and A. Rosenblueth (1949) The Supersensitivity of Denervated Structures, Macmillan Publishing Co., Inc., New York.
Chu, N. S., and F. E. Bloom (1974) The catecholamine-containing neurons in the cat dorsolateral pontine tegmentum: Distribution of the cell bodies and some axonal projections. Brain Res. 66: 1-21.

Clark, D., M. Blair, J. C. King, and R. M. Kostrezewa (1979) Loss of nerve cell bodies in caudal locus coeruleus following treatment of neonates with 6-hydroxydopa. Brain Res. 46: 331-336.

Clark, D. W., R. Laverty, and E. L. Phelan (1972) Long-lasting peripheral and central effects of 6-hydroxydopamine in rats. Br. J. Pharmacol. 44: 233-243.

Daniels, J. D., J. L. Norman, and J. D. Pettigrew (1978) Development of single unit responses in kitten lateral geniculate nucleus. J. Neurophysiol. 41: 1373.

Daw, N. W., and R. K. Rader (1981) Effects of intracortical infusion of 6-OHDA on monocular and directional deprivation. Invest. Ophthalmol. Vis. Sci. (Suppl.) 20: 72.

Descarries, L., K. C. Watkins, and Y. Lapierre (1977) Noradrenergic axon terminals in the cerebral cortex of the rat. III. Topometric ultrastructural analysis. Brain Res. 133: 197-222.

Doba, N., and D. J. Reis (1974) Role of central and peripheral adrenergic mechanisms in neurogenic hypertension produced by brainstem lesions in rats. Circ. Res. 34: 293-301.

Fregnac, Y., and M. Imbert (1978) Farly development of visual cortical cells in normal and dark-reared kittens: Relationship between orientation selectivity and ocular dominance. J. Physiol. (Lond.) 278: 27-44.

Guillery, R. W. (1972) Binocular competition in the control of geniculate cell growth. J. Comp. Neurol. 144: 117-130.

Harik, S. I., R. Bradford Duckrow, J. C. LaManna, M. Rosenthal, V. K. Sharma, and S. P. Banerjee (1981) Cerebral compensation for chronic noradrenergic denervation induced by locus ceruleus lesion: Recovery of receptor binding, isoproterenol-induced adenylate cyclase activity, and oxidative metabolism. J. Neurosci. 1: 641-649.

Hubel, D. H., and T. N. Wiesel (1962) Receptive fields, binocular interactions and functional architecture in the cat's visual cortex. J. Physiol. (Lond.) 160: 106-154.

Hubel, D. H., and T. N. Wiesel; (1970) The period of susceptibility to the physiological effects of unilateral eye closure in kittens. J. Physiol. (Lond.) 206: 419-436.

Hubel, D. H., T. N. Wiesel, and S. LeVay (1977) Plasticity of ocular dominance columns in monkey striate cortex. Philos. Trans. R. Soc. Lond. (Biol.) 278: 377-409.

Johnston, M. V., M. McKinney, and J. T. Coyle (1981) Neocortical cholinergic innervation: A description of extrinsic and intrinsic components in the rat. Exp. Brain Res. 43: 159-172.

Kasamatsu, T., and J. D. Pettigrew (1979) Preservation of binocularity after monocular deprivation in the striate cortex of kittens treated with 6-hydroxydopamine. J. Comp. Neurol. 185: 139-162.

Kasamatsu, T., J. D. Pettigrew, and M. Ary (1979) Restoration of visual cortical plasticity by local microperfusion of norepinephrine. J. Comp. Neurol. 185: 163-182.

Kasamatsu, T., T. Itakura, and G. Jonsson (1981a) Intracortical spread of exogenous catecholamines: Effective concentration for modifying cortical plasticity. J. Pharmacol. Exp. Ther. 217: $841-850$.

Kasamatsu, T., J. D. Pettigrew, and M. Ary (1981b) Cortical recovery from effects of monocular deprivation: Acceleration with norepinephrine and suppression with 6-hydroxydopamine. J. Neurophysiol. 45: 254-266.

Keller, R., A. Oke, I. Mefford, and R. N. Adams (1976) Liquid chromatographic analysis of catecholamines: Routine assay for regional brain mapping. Life Sci. 19: 995-1004.

Kostrzewa, R. M., and R. E. Garey (1976) Effects of 6-hydroxydopa on noradrenergic neurons in the developing rat brain. J. Pharmacol. Exp. Ther. 197: 105-118.

Kratz, K. E., P. D. Spear, and D. C. Smith (1976) Posteritical- 
period reversal of effects of monocular deprivation on striate cortex cells in the cat. J. Neurophysiol. 39: 501-511.

Krnjevic, K., and J. W. Phillis (1963) Iontophoretic studies of neurones in the mammalian cerebral cortex. J. Physiol. (Lond.) 165: 274-304.

LaManna, J. C., S. I. Harik, A. I. Light, and M. Rosenthal (1981) Norepinephrine depletion alters cerebral oxidative metabolism in the "active" state. Brain Res. 204: 87-101.

LeVay, S., T. N. Wiesel, and D. H. Hubel (1980) The development of ocular dominance columns in normal and visually deprived monkeys. J. Comp. Neurol. 191: 1-52.

Levitt, P., and R. Y. Moore (1979) Development of the noradrenergic innervation of neocortex. Brain Res. 162: 243-259.

Lidov, H. G. W., and M. E. Molliver (1982) The structure of cerebral cortex in the rat following prenatal administration of 6-hydroxydopamine. Dev. Brain Res. 3: 81-108.

McMahon, D. (1974) Chemical messengers in development: A hypothesis. Science 185: 1012-1021.

Minneman, K. P., M. D. Dibner, B. B. Wolfe, and P. B. Molinoff (1979) $\beta-1$ and $\beta$-2 Adrenergic receptors in rat cerebral cortex. Science 204: 866-868.

Morrison, J. H., M. E. Molliver, R. Grzanna, and J. T. Coyle (1981) The intracortical trajectory of the coeruleo-cortical projection in the rat: A tangentially organized cortical afferent. Neuroscience 6: 139-158.

Movshon, J. A., and R. C. Van Sluyters (1981) Visual neural development. Annu. Rev. Psychol. 32: 477-522.

Olson, C. R., and R. D. Freeman (1975) Progressive changes in kitten striate cortex during monocular vision. J. Neurophysiol. 38: 26-32.

Olson, C. R., and R. D. Freeman (1980) Rescaling of the retinal map of visual space during the growth of the kitten's eye. Brain Res. 186: 55-65.

Parnavelas, J. G., and M. E. Blue (1982) The role of the noradrenergic system on the formation of synapses in the visual cortex of the rat. Dev. Brain Res. 3: 140-144.

Reader, T. A., Z. Dechamplain, and H. Jasper (1976) Catecholamines released from cerebral cortex in the cat: Decrease during sensory stimulation. Brain Res. 111: 95-108.

Reader, T. A., P. Masse, and J. de Champlain (1979) The intracortical distribution of norepinephrine, dopamine and serotonin in the cerebral cortex of the cat. Brain Res. 177: 499-513.

Sachs, C. (1973) Development of the blood-brain barrier for 6hydroxydopamine. J. Neurochem. 20: 1753-1760.

Sachs, C., and G. Jonsson (1975) Effects of 6-hydroxydopamine on central noradrenaline neurons during ontogeny. Brain Res. 99: 277-291.

Schlumpf, M., W. J. Shoemaker, and F. E. Bloom (1980) Innervation of embryonic rat cerebral cortex by catecholaminecontaining fibers. J. Comp. Neurol. 192: 361-376.

Sporn, J. R., B. B. Wolfe, T. R. Harden, T. Kendall, and P. B. Molinoff (1977) Supersensitivity in rat cerebral cortex: Preand post-synaptic effects of 6-hydroxydopamine at noradrenergic synapses. Mol. Pharmacol. 13: 1170-1180.

Tork, I., and S. Turner (1981) Histochemical evidence for a catecholaminergic (presumably dopaminergic) projection form the ventral mesencephalic tegmentum to visual cortex in the cat. Neurosci. Lett. 24: 215-219.

Ungerstedt, U. (1968) 6-Hydroxydopamine induced degeneration of central monoamine neurons. Eur. J. Pharmacol. 5: 107-110.

Uretsky, N. J., and L. L. Iverson (1970) Effects of 6-hydroxydopamine on catecholamine containing neurons in the rat brain. J. Neurochem. 17: 269-278.

Wendlandt, S., T. J. Crow, and R. V. Stirling (1977) The involvement of the noradrenergic system arising from the locus coeruleus in the postnatal development of the cortex in rat brain. Brain Res. 125: 1-9. 